\title{
8-2012
}

\section{Adaptive volume penalization for ocean modeling}

\author{
Shanon M. Reckinger \\ Fairfield University, shanon.reckinger@fairfield.edu
}

Oleg V. Vasilyev

Baylor Fox-Kemper

Follow this and additional works at: https://digitalcommons.fairfield.edu/engineering-facultypubs Copyright 2012 Springer.

Permission has been granted to archive the author post-print. The final publication is available at http://link.springer.com/article/10.1007/s10236-012-0555-3.

\section{Peer Reviewed}

\section{Repository Citation}

Reckinger, Shanon M.; Vasilyev, Oleg V.; and Fox-Kemper, Baylor, "Adaptive volume penalization for ocean modeling" (2012). Engineering Faculty Publications. 114.

https://digitalcommons.fairfield.edu/engineering-facultypubs/114

\section{Published Citation}

Reckinger, Shanon M., Oleg V. Vasilyev, and Baylor Fox-Kemper. "Adaptive volume penalization for ocean modeling." Ocean Dynamics 62, no. 8 (August 2012): 1201-1215.

This item has been accepted for inclusion in DigitalCommons@Fairfield by an authorized administrator of DigitalCommons@Fairfield. It is brought to you by DigitalCommons@Fairfield with permission from the rightsholder(s) and is protected by copyright and/or related rights. You are free to use this item in any way that is permitted by the copyright and related rights legislation that applies to your use. For other uses, you need to obtain permission from the rights-holder(s) directly, unless additional rights are indicated by a Creative Commons license in the record and/or on the work itself. For more information, please contact digitalcommons@fairfield.edu. 
Noname manuscript No.

(will be inserted by the editor)

\title{
Adaptive Volume Penalization for Ocean Modeling
}

\author{
Shanon M. Reckinger - Oleg V. \\ Vasilyev • Baylor Fox-Kemper
}

Received: date / Accepted: date

\begin{abstract}
The development of various volume penalization techniques for use in modeling topographical features in the ocean is the focus of this paper. Due to the complicated geometry inherent in ocean boundaries, the stair-step representation used in the majority of current global ocean circulation models causes accuracy and numerical stability problems. Brinkman penalization is the basis for the methods developed here and is a numerical technique used to enforce no-slip boundary conditions through the addition of a term to the governing equations. The second aspect to this proposed approach is that all governing equations are solved on a non-uniform, adaptive grid through the use of the Adaptive Wavelet Collocation Method. This method solves the governing equations on temporally and spatially varying meshes, which allows higher effective resolution to be obtained with less computational cost. When penalization methods are coupled with the Adaptive Wavelet Collocation Method,

S. M. Reckinger

Fairfield University

School of Engineering, Department of Mechanical Engineering

1073 North Benson Rd.

Fairfield, CT 06824

Tel.: +001-203-254-4000 ext. 2527

E-mail: shanon.reckinger@fairfield.edu

O. V. Vasilyev

University of Colorado at Boulder

Department of Mechanical Engineering, UCB 427

Boulder, CO 80309

Tel.: +001-303-492-4717

E-mail: oleg.vasilyev@colorado.edu

B. Fox-Kemper

University of Colorado at Boulder

Department of Cooperative Institute for Research in Environmental Sciences (CIRES)

Department of Atmospheric and Oceanic Sciences, UCB 216

Boulder, CO 80309

Tel.: +001-303-492-0532

E-mail: bfk@colorado.edu
\end{abstract}


the flow near the boundary can be well resolved. It is especially useful for simulations of boundary currents and tsunamis, where flow near the boundary is important. This paper will give a thorough analysis of these methods applied to the shallow water equations, as well as some preliminary work applying these methods to volume penalization for bathymetry representation for use in either the non-hydrostatic or hydrostatic primitive equations.

Keywords Immersed Boundary Methods · Shallow Water Equations · Adaptive Mesh Refinement · Wavelet Collocation · Complex Geometry

\section{Introduction}

In recent years, substantial progress has been made in the development of numerical methods used in ocean modeling. The newly developed ocean models have started incorporating more sophisticated numerical methods, such as finite element methods, spectral methods and finite volume methods, which are solved on adaptive and/or unstructured grids (Chen et al 2003; Danilov et al 2004; Fringer et al 2006; Iskandarani et al 2003; Lynch et al 1996; Marshall et al 1997; Herrnstein et al 2005; Pain et al 2005; Popinet and Rickard 2007; White 2007). Structured grid models have been the mainstay of ocean modeling for over 50 years with compromises for complex bathymetry (Adcroft et al 1997) and unstructured grid models are beginning to allow for much more complex bathymetry. However, Brinkman penalization is an approach that can be used for complex bathymetry regardless of grid structure, and it can even be used for bathymetry that evolves in time (e.g., sedimentation, turbines, seafloor earthquakes). Since the ocean is a strongly coupled multiscale system, accurate representation of the entire range of scales calls for state of the art numerical methods and techniques. To handle this immense range of spatial and temporal scales, there is a need to dynamically resolve significant structures. The proposed approach not only solves the ocean governing equations on an on-the-fly adaptive grid, but also provides a computationally efficient technique for representing complex boundaries.

The focus of this paper is to present a complete approach for modeling topographical features in the ocean using various forms of volume penalization. Modeling complex boundaries is a pressing issue in the field of ocean modeling. The majority of current ocean models use body-fitted meshes, which are expensive and often have stability issues when representing boundaries with complicated geometry (Collins et al 2006). Immersed boundary methods are well known for the their efficient implementation of solid boundaries of arbitrary complexity on fixed non-body conformal Cartesian grids (Mittal and Iaccarino 2005; Peskin 2002). Immersed boundary techniques are rarely used in ocean modeling (Tseng and Fersiger 2003). Brinkman penalization, a type of immersed boundary method, has been used in many engineering problems to simulate the presence of arbitrarily complex solid obstacles and boundaries (Arquis and Caltagirone 1984). This volume penalization technique is a way to enforce boundary conditions to a specified precision without changing 
the numerical method or grid used in solving the equations. Its main idea is to model arbitrarily complex solid obstacles as porous media with porosity, $\phi$, and permeability approaching zero. The main advantage of Brinkman penalization, when compared to other penalization methods, is that the error can be estimated rigorously and controlled via a penalization parameter (Angot et al 1999). This allows for complete control of the accuracy of the boundary conditions. Additionally, it can be shown that the penalized equations converge to the exact solution in the limit as the penalization parameter tends to zero (Angot 1999). The work of Adcroft and Marshall (1998) shows that implementing no-slip boundary conditions can be error-prone for traditional approaches, while Adcroft et al (1997) demonstrate the importance of capturing topographic features that are not neatly captured by the edges of finite-volume cells. Brinkman penalization automatically handles both cases, for arbitrary bottom slope and shelf orientation.

Immersed boundary methods have been developed for incompressible flows and more recently have been extended to compressible flows (Liu and Vasilyev 2007). Both of these formulations have been adapted to be used on the ocean governing equations (including the shallow water equations and the hydrostatic and non-hydrostatic primitive equations). This paper will be primarily focusing on the extension of these methods to the shallow water equations, but will present preliminary results of applying Brinkman penalization to the primitive equations.

In order to model complex geometries, a non-uniform, adaptive mesh is ideal. For many adaptive models, the main challenge is grid generation. Not only is grid generation difficult, but the process used is often trial and error. It is also computationally expensive. Additionally, many other adaptive grid models require grid generation at every time step. Ideally, the grid should follow the structures in the flow, in addition to adapting to the complicated curves of the bathymetry. In this work, this is done by the combination of two mathematical approaches: Brinkman penalization (Arquis and Caltagirone 1984) and the Adaptive Wavelet Collocation Method (Vasilyev 2003; Vasilyev and Bowman 2000; Vasilyev and Kevlahan 2002). The Adaptive Wavelet Collocation Method efficiently resolves localized flow structures in complicated geometries, while the Brinkman penalization efficiently implements arbitrarily complex solid boundaries. Brinkman penalization is a natural technique to use on problems with adaptive methods, because the adaptive meshes will ensure adequate resolution at the boundary. This is especially important for problems where the physics near the boundaries play a considerable role in the overall features of the flow.

The hybrid wavelet collocation - Brinkman penalization method has been previously investigated for three cases: incompressible Navier-Stokes equations both in vorticity (Vasilyev and Kevlahan 2002) and primitive variable formulations (Kevlahan et al 2000; Kevlahan and Vasilyev 2005), and compressible Navier-Stokes equations in primitive variable formulation (Liu and Vasilyev 2007). High Reynolds number flows can be simulated while greatly reducing the number of wavenumber modes and controlling the error. The computa- 
tional cost of the wavelet method is independent of the dimensionality of the problem. It is $O(\mathcal{N})$, where $\mathcal{N}$ is the total number of wavelets actually used. The Adaptive Wavelet Collocation Method uses second generation wavelets, which allows the order of the method to be variable. Also, the method is easily applied in both two and three dimensions.

In the following sections, the Adaptive Wavelet Collocation Method will be discussed, followed by an explanation of the extension of the compressible formulation of Brinkman penalization to be used on the shallow water equations. It was found that the application of the existing Brinkman penalization (developed for the compressible equations) on the shallow water equations was not sufficient and therefore several modifications of the Brinkman penalization method were made and fully tested. Finally, some preliminary work is presented on the development of a volume penalization method to be used on the hydrostatic and non-hydrostatic primitive equations for both no-slip and slip boundary conditions.

\section{Adaptive Wavelet Collocation Method}

The Adaptive Wavelet Collocation Method is a general method for the solution of a large class of linear and nonlinear partial differential equations (Vasilyev and Bowman 2000; Vasilyev 2003; Vasilyev and Kevlahan 2005; Regele and Vasilyev 2009). The method has already been successfully applied in wide range of fluid mechanics problems, e.g., Vasilyev et al 1997; Vasilyev and Kevlahan 2002; Kevlahan et al 2007; Reckinger et al 2010; Schneider and Vasilyev 2010. In this section, the methodology is briefly reviewed.

The benefit of using wavelets is that they are localized in both space and time. They are ideal for use in complex flows where localized structures exist in the solution. The wavelet collocation method takes advantage of wavelet compression properties. Functions with localized structures or regions with sharp transitions are well compressed using wavelet decomposition. This compression is achieved by keeping only the wavelets with coefficients that are greater than an a priori threshold parameter. This allows high resolution computations to be carried out only in the regions where it is necessary. It also allows a solution to be obtained on a near optimal grid for a given accuracy. Figure 1 shows an example of a simulation of the 2010 Chile Tsunami using the Adaptive Wavelet Collocation Method to solve the shallow water equations with real variable bathymetry. The right side of the figure shows that the grid is localized near the tsunami and near all the continental boundaries.

Any function $u(\mathbf{x})$ in an $n$-dimensional space can be decomposed as (Chui 1997; Daubechies 1992; Mallat 1998)

$$
u(\mathbf{x})=\sum_{\mathbf{k} \in \mathcal{K}^{0}} c_{\mathbf{k}}^{0} \phi_{\mathbf{k}}^{0}(\mathbf{x})+\sum_{j=0}^{+\infty} \sum_{\mu=1}^{2^{n}-1} \sum_{\mathbf{l} \in \mathcal{L}^{\mu, j}} d_{1}^{\mu, j} \psi_{\mathbf{1}}^{\mu, j}(\mathbf{x}),
$$




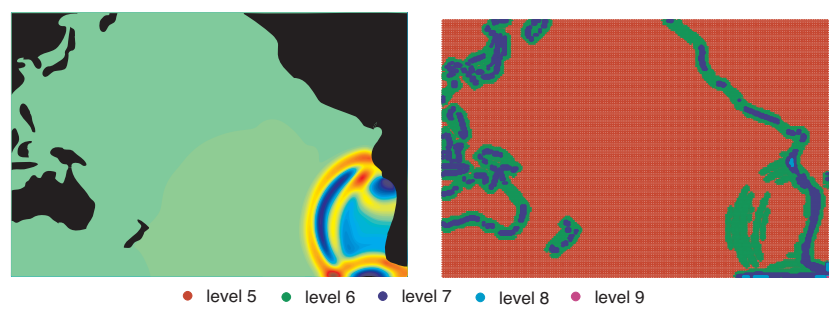

Fig. 1 Preliminary results from 2010 Chile Tsunami simulation, with sea surface height on the left and the adaptive grid (colored by level) on the right using the Adaptive Wavelet Collocation Method.

where $\phi_{\mathbf{k}}^{0}(\mathbf{x})$ are scaling functions on the lowest level of resolution and $\psi_{1}^{\mu, j}(\mathbf{x})$ are the wavelet basis functions. Also, $c_{\mathbf{k}}^{0}$ and $d_{1}^{\mu, j}$ are the scaling and wavelet coefficients, respectively. The wavelet coefficients, $d_{1}^{\mu, j}$, are small except near areas with large gradients. Equation 1 can be decomposed into two terms whose wavelet coefficients are above and below a chosen threshold parameter $\epsilon$,

$$
u(\mathbf{x})=u_{\geq}(\mathbf{x})+u_{<}(\mathbf{x}),
$$

where

$$
\begin{aligned}
& u_{\geq}(\mathbf{x})=\sum_{\mathbf{k} \in \mathcal{K}^{0}} c_{\mathbf{k}}^{0} \phi_{\mathbf{k}}^{0}(\mathbf{x})+\sum_{j=0}^{+\infty} \sum_{\mu=1}^{2^{n}-1} \sum_{\substack{1 \in \mathcal{L}^{\mu, j} \\
\left|d_{1}^{\mu, j}\right| \geq \epsilon\|u\|}} d_{\mathbf{l}}^{\mu, j} \psi_{\mathbf{1}}^{\mu, j}(\mathbf{x}), \\
& u_{<}(\mathbf{x})=\sum_{j=0}^{+\infty} \sum_{\mu=1}^{2^{n}-1} \sum_{\substack{\mathbf{1} \in \mathcal{L}^{\mu, j} \\
\left|d_{1}^{\mu, j}\right|<\epsilon\|u\|}} d_{1}^{\mu, j} \psi_{\mathbf{1}}^{\mu, j}(\mathbf{x}),
\end{aligned}
$$

Donoho (1992) was able to show that for a regular function the error is bounded as

$$
\left\|u(\mathbf{x})-u_{\geq}(\mathbf{x})\right\| \leq C_{1} \epsilon\|u\|,
$$

which means that the number of grid points needed to solve a numerical problem can be significantly reduced while still retaining a prescribed level of accuracy determined by the threshold parameter $\epsilon$.

In the wavelet collocation method there is a one-to-one correspondence between grid points and wavelets. This makes calculation of nonlinear terms simple, and allows the grid to adapt automatically to the solution at each time step by adding or removing wavelets. In addition to the points with significant wavelet coefficients, several other checks are performed to ensure the resolution is sufficient for the given simulation. The way the method works is, at the beginning of each time step, the wavelet coefficients are calculated. Wavelets with significant coefficients are identified. Next, to account for the evolution of the solution over time, the nearest neighbor wavelet coefficients 
in position and scale are also added (Liandrat and Tchamitchian 1990). After these significant and adjacent points are kept, the wavelets that are below the threshold $\epsilon$ and are not in the adjacent zone are removed. It can be shown that the $L_{\infty}$ error for this approximation is bounded by $\epsilon$. This allows the grid to automatically follow the evolution of the solution. Then, reconstruction points are added, which are points needed to compute the wavelet transforms. Lastly, ghost points are added, these are points needed to calculate spatial derivatives. The spatial derivatives are calculated using finite differences. Since this method uses second generation wavelets (Sweldens 1998), the order of the wavelet (and also finite difference) can be easily varied.

Figure 2 shows a one-dimensional example of a solution (top) and its adaptive grid (bottom). The vertical lines show the magnitude of the wavelet coefficients at each location in space for each level of resolution. It is clear that at the location in the center of the $x$-axis where the solution has a sharp gradient, there is localized refinement on the grid.
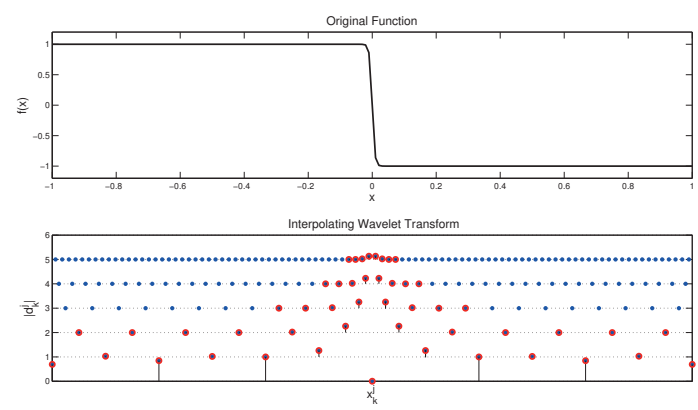

Fig. 2 A one dimensional example of grid adaptation using the Adaptive Wavelet Collocation Method.

There are some additional computational costs associated with the use of the adaptive multi-resolution wavelet method. Currently, the cost per grid point is approximately three to five times greater than the cost of a standard non-adaptive method. However, in cases of large compressions (Kevlahan and Vasilyev 2005) (up to $10^{3}$ ), the compression greatly outweighs this cost. There is also some memory savings associated with using adaptive methods, which allows higher resolution simulations with the same computational resources.

In summary, the dynamically Adaptive Wavelet Collocation Method is an adaptive, variable order method for solving partial differential equations with localized structures that change their location and scale in space and time. Since the computational grid automatically adapts to the solution (in position and scale), we do not have to know a priori where the regions of high gradients or structures exist. 


\section{Brinkman Penalization for Shallow Water Model}

The shallow water equations are mathematically similar to the compressible Euler equations. A compressible formulation of Brinkman penalization has been developed by Liu and Vasilyev (2007). This compressible form is extended to the shallow water equations,

$$
\begin{gathered}
\frac{\partial \eta}{\partial t}=-\left[1+\left(\frac{1}{\phi}-1\right) \chi\right] \nabla \cdot(\eta \mathbf{u}), \\
\frac{\partial \mathbf{u}}{\partial t}+\mathbf{u} \cdot \nabla \mathbf{u}+\frac{1}{R o} f \hat{\mathbf{k}} \times \mathbf{u}=-\frac{1}{F r^{2}} \nabla \eta-\frac{\chi}{\eta_{\text {pen }}} \mathbf{u}
\end{gathered}
$$

where the Rossby number, $R o=U / L f$, the Froude number, $F r=U / \sqrt{g H}$, the Brinkman penalization parameter, $\eta_{\text {pen }} \ll 1$, the porosity parameter, $\phi \ll 1$, and,

$$
\chi(\mathbf{x}, t)= \begin{cases}1 & \text { if } \mathbf{x} \in O_{i}(\mathbf{x}) \\ 0 & \text { otherwise }\end{cases}
$$

which is called a masking function. $O_{i}(\mathbf{x})$ is any obstacle, or, in the case of shallow water ocean simulations, is the continental boundaries.

Analysis of the equations and numerical testing show that there are three main differences between the shallow water equations and the compressible equations. These differences result in a different treatment of the penalization parameters and the numerical set up compared to the compressible form.

The following analysis describes these differences, while also considering amplitude and phase error analysis for the case of gravity wave propagation in the small amplitude limit.

\subsection{Wave Speeds}

One of the assumptions that is made when performing the error analysis for compressible Brinkman penalization is that the speed of sound is the same in the fluid region and porous media region (Liu and Vasilyev 2007). For the penalized shallow water equations, the gravity wave speed is different in the fluid versus the porous media. Consider the one dimensional shallow water equations,

$$
\frac{\partial \mathbf{u}}{\partial t}+\mathbf{A} \frac{\partial \mathbf{u}}{\partial x}=0
$$

where $\mathbf{u}=(\eta, \eta u)^{T}$ is the vector of conservative, dimensional variables and the Jacobian matrix is

$$
\mathbf{A}=\left[\begin{array}{cc}
0 & \frac{1}{\phi} \\
g \eta-\frac{(\eta u)^{2}}{\eta^{2}} & \frac{2(\eta u)}{\eta}
\end{array}\right] .
$$


Note that when $\phi=1$, the equations reduce to the traditional shallow water equations. Therefore, $\phi=1$ represents the fluid case, while all other cases are for the porous media. The eigenvalues of $\mathbf{A}$ are

$$
\lambda=u \pm \sqrt{u^{2}-\frac{1}{\phi}\left(u^{2}-g \eta\right)} .
$$

Assuming that $u / \sqrt{g H}=O(\varepsilon)$ and $\eta / H=1+O(\varepsilon)$, where $H$ is the mean depth of the ocean and $\varepsilon$ is some small perturbation $(\varepsilon \ll 1)$, the eigenvalues become

$$
\lambda=u \pm \sqrt{\frac{g H}{\phi}} .
$$

When $\phi=1$, the eigenvalues are $u \pm \sqrt{g H}$ as expected for the shallow water gravity wave speed. However, for any other $\phi$ value, the eigenvalues are different. Inside the porous media, where $\phi \ll 1$, the gravity wave speed is much larger than in the fluid region. The implementation issues related to these difficulties are discussed in Section 4.2.

\subsection{Impedance}

Some of the properties associated with the classical theory of acoustics (Blackstock 2000) are used in the development of the compressible formulation of Brinkman penalization (Liu and Vasilyev 2007). Consider the plane wave reflection and transmission at the interface between two different media. To model the one dimensional problem of wave propagation from a fluid into a porous media, it can be thought of as a sudden change in cross-sectional area (Liu and Vasilyev 2007). From acoustics theory, the acoustic impedance at a given surface is the ratio of the surface-averaged acoustic pressure to the fluid volume velocity,

$$
Z=\frac{\rho c}{S}
$$

where $\rho$ is density, $c$ is gravity wave speed in the fluid $(c=\sqrt{g H})$, and $S$ is the cross-sectional area. In order to have most of the wave reflected, the obstacle's acoustic impedance needs to be sufficiently large, which is the basis for the Impedance Mismatch Method (Chung 1995). For the shallow water equations, since the gravity wave speed is also a function of $\phi$, the impedance becomes

$$
Z=\frac{\rho c}{\phi^{3 / 2}},
$$

which is a higher impedance than what was found for the compressible Brinkman penalization formulation, which was $Z=\rho c / \phi$ (Liu and Vasilyev 2007). This allows for a slightly lower range of reasonable $\phi$ values for negligible wave transmission in the shallow water case compared to the compressible case. This is one advantage of the shallow water formulation of Brinkman penalization. 


\subsection{Analogy to Euler Equations}

The compressible equations in the isothermal limit yield similar results as the shallow water equations. The 1D Euler equations with either isothermal or adiabatic conditions (i.e. $\partial P / \partial x=c^{2} \partial \rho / \partial x$ ), reduce to

$$
\begin{array}{r}
\frac{\partial \rho}{\partial t}+\frac{1}{\phi} \frac{\partial \rho u}{\partial x}=0 \\
\frac{\partial \rho u}{\partial t}+\frac{\partial}{\partial x}(\rho u u)+c^{2} \frac{\partial \rho}{\partial x}=-\frac{1}{\eta_{\text {pen }}} u .
\end{array}
$$

These assumptions eliminate the energy equation. Unlike the full compressible form of Brinkman penalization, this form (like the shallow water equation form) only has two penalization parameters, $\phi$ and $\eta_{\text {pen }}$.

Similar analysis as Section 3.1 yields the following eigenvalues:

$$
\lambda=u \pm \frac{c}{\sqrt{\phi}} .
$$

This analysis highlights the similarities between the shallow water and compressible equations. Elimination of the energy equation effectively removes entropy waves leaving only two acoustic waves in the equations, with a subsequent change of wave speed inside the Brinkman zone.

\subsection{Amplitude and Phase Errors by Asymptotic Analysis}

The use of asymptotic analysis provides a way to estimate the amplitude and phase errors associated with the penalized shallow water equations. In addition, by looking at the equations in different asymptotic limits, information about the behavior of the system is obtained from a rigorous mathematical viewpoint. The "ocean region" simply refers to the part of the numerical domain where the shallow water equations are being solved. The "continental region" refers to the part of the numerical domain where the penalized shallow water equations are solved. The following analysis assumes small amplitude waves in the ocean region and is similar to the analysis performed for the penalized compressible equations (Liu and Vasilyev 2007).

\subsubsection{Asymptotic Analysis for the Ocean Region}

The ocean region variables are written as

$$
\begin{gathered}
\eta_{\mathrm{o}}=1+\varepsilon \eta_{\mathrm{o}}^{\prime}, \\
u_{\mathrm{o}}=\varepsilon u_{\mathrm{o}}^{\prime},
\end{gathered}
$$

where $\varepsilon \ll 1$, small perturbations from the mean. Sea surface height is the mean ocean depth plus the sea surface height perturbations, where the velocity is only a function of the velocity perturbations. If Equations 18 and 19 are 
substituted into Equations 6 and 7 in the limit of large Rossby and Froude numbers, and only the leading perturbation terms are retained, the result is a wave equation,

$$
\begin{aligned}
\frac{\partial^{2} \eta_{\mathrm{o}}^{\prime}}{\partial t^{2}} & =\frac{\partial^{2} \eta_{\mathrm{o}}^{\prime}}{\partial x^{2}}, \\
\frac{\partial^{2} u_{\mathrm{o}}^{\prime}}{\partial t^{2}} & =\frac{\partial^{2} u_{\mathrm{o}}^{\prime}}{\partial x^{2}} .
\end{aligned}
$$

Thus, in the fluid or ocean region, the equations reduce to a wave equation.

\subsubsection{Asymptotic Analysis for Continental Region}

For the continental region, the variables can be written as

$$
\begin{gathered}
\eta_{\mathrm{c}}=1+\varepsilon \eta_{\mathrm{c}}^{\prime}, \\
u_{\mathrm{c}}=\varepsilon \eta_{\text {pen }} u_{\mathrm{c}}^{\prime} .
\end{gathered}
$$

The leading perturbation terms in this case are different from the ocean region because of the strong Brinkman damping term in the momentum equation. If Equations 22 and 23 are substituted into Equations 6 and 7 and only the leading order terms are retained, the result is a diffusion equation,

$$
\begin{aligned}
\frac{\partial \eta_{\mathrm{c}}^{\prime}}{\partial t} & =\alpha \frac{\partial^{2} \eta_{\mathrm{c}}^{\prime}}{\partial x^{2}} \\
\frac{\partial u_{\mathrm{c}}^{\prime}}{\partial t} & =\alpha \frac{\partial^{2} u_{\mathrm{c}}^{\prime}}{\partial x^{2}},
\end{aligned}
$$

where $\alpha=\eta_{\mathrm{pen}} / \phi$. Thus, in the porous media or continental region, the equations reduce to a diffusion equation.

\subsubsection{Asymptotic Analysis for Boundary Layer}

The asymptotic analysis for the ocean and continental region is valid only away from the interface, because of the length and magnitude scales of the perturbations used. Therefore, the boundary layer region needs to be analyzed separately. The boundary layer variables can be written as

$$
\begin{aligned}
& \eta_{\mathrm{bl}}=1+\varepsilon \eta_{\mathrm{bl}}^{\prime}, \\
& u_{\mathrm{bl}}=\varepsilon \eta_{\mathrm{pen}} u_{\mathrm{bl}}^{\prime} .
\end{aligned}
$$

If Equations 26 and 27 are substituted into Equations 6 and 7 and only the leading order terms are kept, the result is a diffusion equation,

$$
\begin{aligned}
\frac{\partial \eta_{\mathrm{bl}}^{\prime}}{\partial t} & =\alpha \frac{\partial^{2} \eta_{\mathrm{bl}}^{\prime}}{\partial x^{2}} \\
\frac{\partial u_{\mathrm{bl}}^{\prime}}{\partial t} & =\alpha \frac{\partial^{2} u_{\mathrm{bl}}^{\prime}}{\partial x^{2}} .
\end{aligned}
$$


This is where the penalized shallow water equations differ greatly from the penalized compressible equations. For the compressible equations, there is a boundary layer region that provides a natural transition between the two other asymptotic solutions (Liu and Vasilyev 2007). That is, in addition to the fluid region being governed by the acoustic wave equation and the porous media region being governed by a diffusion equation, there exists a boundary layer region in between that is governed by a diffusive wave equation. This boundary layer region does not exist in the penalized shallow water equations. As demonstrated in the above analysis, the boundary layer region is the same as the continental region, leaving no natural, mathematical transition. As a result, in order to ensure numerical stability, the shallow water formulation requires a numerical boundary layer, which needs to be resolved. This makes it slightly more computationally expensive compared to the compressible formulation. Note that the no-slip boundary conditions along the coastal line are already an approximation, since the depth of the ocean floor gradually decreases, thus numerically smears no-slip boundary conditions, which may even result in a more accurate representation of the coastal boundary conditions.

\section{Numerical Experiments and Validation of Method}

\subsection{Test Setup}

A one dimensional test case is used to verify convergence of this new Brinkman penalization technique for the shallow water equations. A $1 \mathrm{D}$ normal wave is initialized for the sea surface height. The velocity is initialized to zero, as shown in Figure 3. The wave splits and propagates to the east and west. On the east
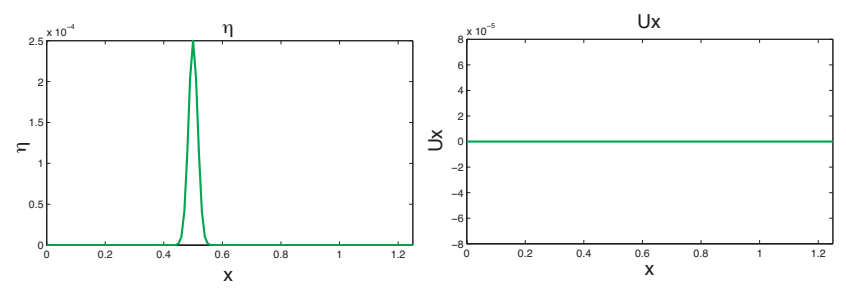

Fig. 3 Example of initial conditions for a $1 \mathrm{D}$ wave.

side, it hits a Brinkman zone and on the west side, it hits a conventional boundary wall, where no-penetration boundary conditions are enforced. This allows for comparison between the two methods. After complete reflection, the solution is shown in Figure 4. 

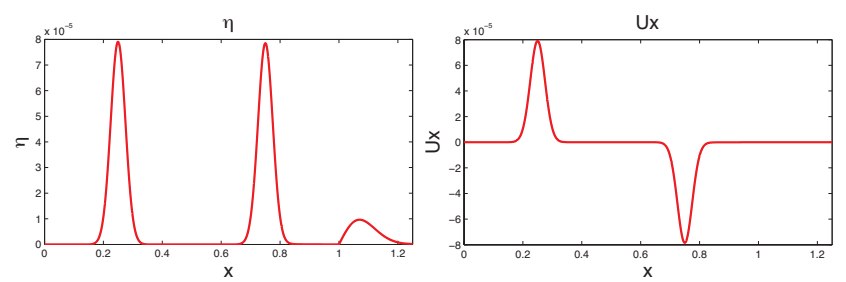

Fig. 4 Example of a 1D wave after complete reflection.

\subsection{Parameter Study}

While there are two parameters specific to the compressible Brinkman penalization formulation, $\phi$ and $\eta_{\text {pen }}$, there are two additional parameters to consider with the shallow water formulation. The first additional parameter is $\delta_{\text {pen }}$, which controls how sharp the transition from land to water is at the Brinkman boundary. This parameter is required in the definition of the masking function, $\chi$, which was stated in general for any solid boundary in Equation 8. For a domain with solid straight boundaries on either side of the horizontal domain, a hyperbolic tangent function can be used to define masking function,

$$
\chi=\frac{1}{4}\left[\tanh \left(\frac{x-x_{\mathrm{O} 1}}{\delta_{\text {pen }}}\right)+\tanh \left(\frac{x-x_{\mathrm{O} 2}}{\delta_{\text {pen }}}\right)\right]^{2},
$$

where $x_{\mathrm{O} 1}$ and $x_{\mathrm{O} 2}$ are the locations of the Brinkman zone boundaries (or where $\chi=1)$. Therefore, when $x_{\mathrm{O} 2}>x>x_{\mathrm{O} 1}$, then $\chi=0$ and the fluid equations are solved. When $x_{\mathrm{O} 1}>x$ or $x_{\mathrm{O} 2}<x$, then $\chi=1$ and the penalized equations are solved. The parameter, $\delta_{\text {pen }}$, controls how sharp the transition between 0 and 1 is.

The second additional parameter is the length scale associated with the initial conditions. This could be defined in many ways, but for the $1 \mathrm{D}$ normal wave used for this convergence study it is simply the thickness of the wave. The initial conditions for this case are of the form,

$$
\eta(x, 0)=\eta_{\mathrm{amp}} \exp \left(-\frac{\left(x-x_{0}\right)^{2}}{\sqrt{\delta_{\eta}}}\right),
$$

where $\eta_{\mathrm{amp}}$ is the amplitude of the sea surface height wave, $x_{0}$ is the location of the center of the wave, and $\sqrt{\delta_{\eta}}$ is the thickness of the wave (the length scale of interest). If the wave approaching the Brinkman zone is smoother or sharper, it is going to affect the length scale and the error convergence of the problem. If the thickness of the wave approaching the boundary is smaller, the error will be bigger. If the thickness of the wave approaching the boundary is larger, the error will be smaller.

Both length scales are important to consider when setting the parameters and reproducing the results presented here. However, since $\sqrt{\delta_{\eta}}$ and $\delta_{\text {pen }}$ are both simply length scales of the problem, it is their ratio that is important. 
For all work presented here, these two parameters will be combined into one, $\gamma=\delta_{\text {pen }} / \sqrt{\delta_{\eta}}$. This means a sharp wave approaching a smooth boundary has a similar error to a smooth wave approaching a sharp boundary. For example, in practice, it means that if the shallow water formulation of Brinkman penalization is being used for wind-driven gyres in a basin, there will be a large length scale associated with that phenomenon. If it were being used to represent bathymetry in tsunami simulations, the tsunami wave lengths are a much shorter length scale. Thus, one can get away with a much smoother mask in a wind-drive gyre problem to maintain the same error as a tsunami simulation with a sharper mask.

The parameter relationships are studied in order to be able to control these new and old parameters for different cases. To start, the additional shallow water equation Brinkman parameters $\left(\delta_{\text {pen }}, \sqrt{\delta_{\eta}}\right.$, and the sea surface height initial conditions) are set to the same values used in the compressible Brinkman formulations in order to do a direct comparison. Therefore, Figure 5 shows the convergence for exactly the same conditions that are used in the compressible Brinkman formulation.

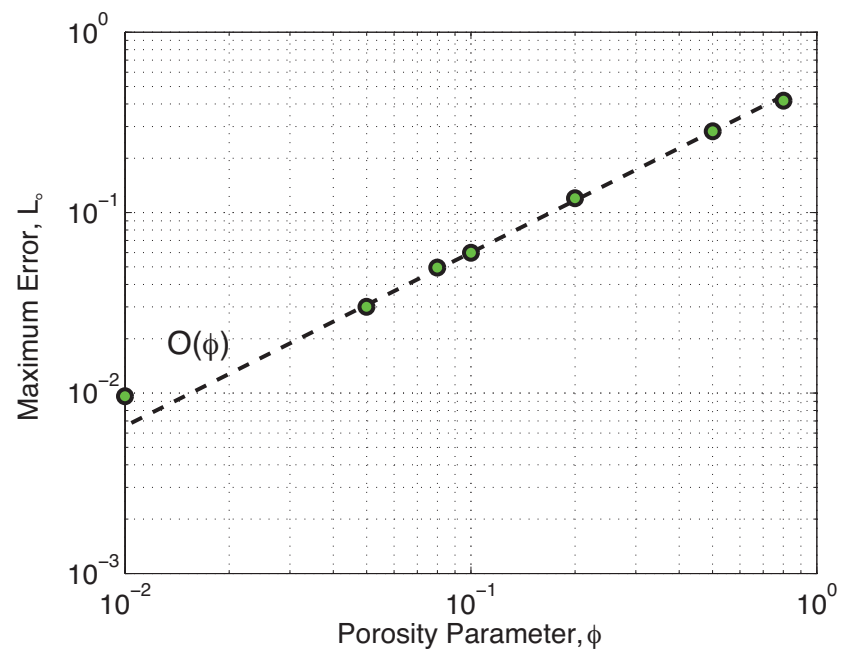

Fig. 5 Plot of max error versus porosity parameter, $\phi$, which demonstrates convergence of approximately $O(\phi)$ (dotted line shows $O(\phi)$ convergence). This convergence uses the same sea surface height incoming wave as in Ref. (Liu and Vasilyev 2007).

Comparing the shallow water formulation to the compressible formulation from Liu and Vasilyev (2007), it is clear that the order of convergence is better for the shallow water case for the more limited range of parameters. For the compressible formulation, the order of convergence is $O\left(\phi^{3 / 4}\right)$, where for the shallow water formulation (for the same initial conditions), the order of convergence is approximately $O(\phi)$. 
The improved convergence is likely due to the stronger impedance inherent in the shallow water Brinkman formulation. Since a larger porosity parameter results in more reflection and less wave transmission, the shallow water formulation converges faster than the compressible formulation. The larger porosity requirement is due to the wave speed change inside the Brinkman region. Smaller values of porosity depend strongly on the resolution and on the thickness of the transition between the fluid and the solid, namely $\delta_{\text {pen }}$. When porosity becomes too small, the increase and jump in the wave speed at the Brinkman boundary cause numerical instabilities and stiffness.

Figure 6 shows the reflected wave with various porosity parameters after complete reflection for the compressible formulation comparison case. Figure 4 shows the sea surface height and velocity solutions after complete reflection for the entire domain, including the Brinkman region. This not only demonstrates how the no-penetration boundary conditions are satisfied in the Brinkman zone, but also shows the exponentially decaying solution for the sea surface height in the Brinkman zone. The small amount of wave transmission is negligible because it is actually multiplied by $\phi$ in the solution. These results match the compressible Brinkman formulation results.

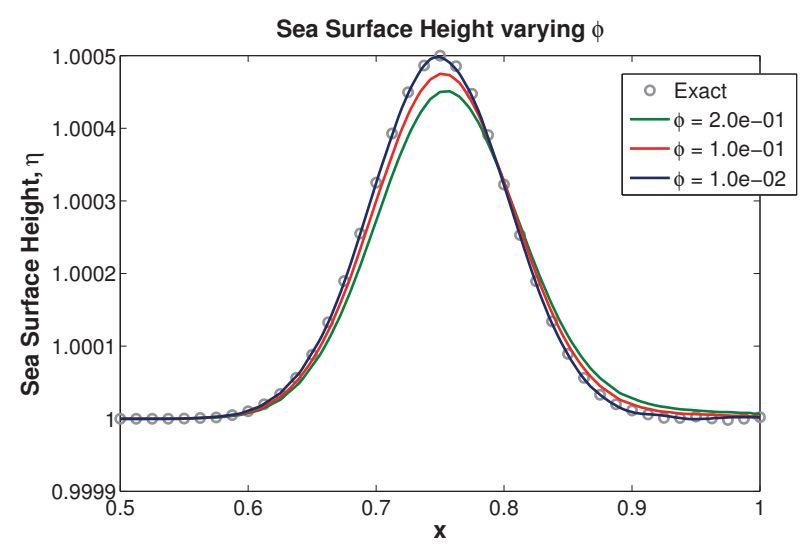

Fig. 6 Plot of sea surface height for various porosity parameters.

In addition to a direct comparison with the compressible Brinkman tests, the following tests are conducted to further understand the influence of the parameters, especially with the $\gamma$ parameter. For these tests, $\delta_{\text {pen }}=1 \times 10^{-4}$, $5 \times 10^{-4}$, and $1 \times 10^{-3}, \eta_{\mathrm{amp}}=2.5 \times 10^{-4}$, and $\delta_{\eta}=5.0 \times 10^{-4}$. This is equivalent to $\gamma=4.46 \times 10^{-3}, 2.23 \times 10^{-2}$, and $4.46 \times 10^{-2}$. These parameter values are chosen to give a large range of feasible $\phi$ values to test. However, even the largest $\delta_{\text {pen }}$ parameter tested is smaller than what would be used for practical applications for the current state of the code. Figure 7 shows convergence results for $\alpha=\eta_{\text {pen }} / \phi=10^{-2}$. 


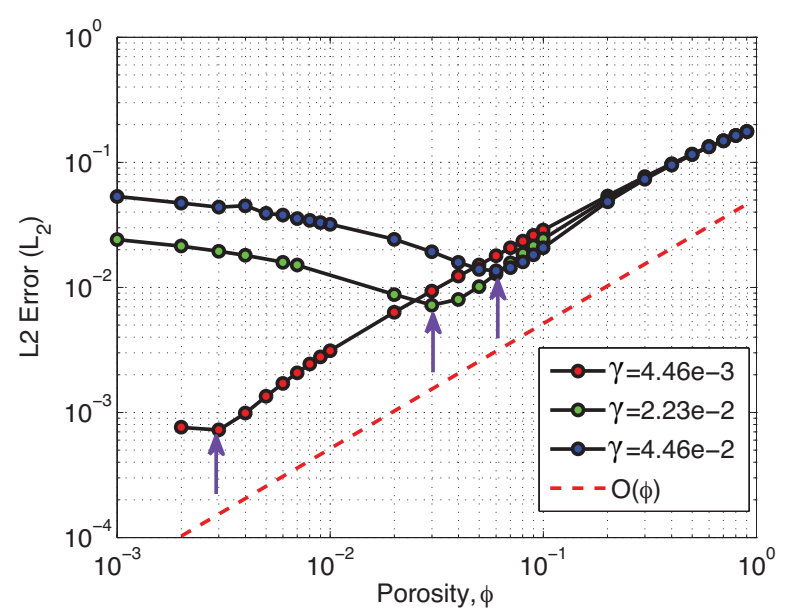

Fig. 7 Plot of L2 error versus porosity parameter, $\phi$ for $\alpha=1 \times 10^{-2}$ and for three different $\gamma$. The purple arrows are pointing to the optimal $\phi$ for each of the given $\gamma$ parameters tested.

It is clear from this convergence study that given a $\gamma$ ratio, as the porosity parameter decreases the error eventually levels off and no longer continues to decrease. The point where the error starts to level off decreases as $\gamma$ decreases. This gives an overall relationship between the three parameters that need to be chosen using the shallow water Brinkman penalization method.

It is important to note that these results are for a fixed ratio of $\phi$ and $\eta_{\text {pen }}$ $\left(\alpha=\eta_{\text {pen }} / \phi=10^{-2}\right)$. It was found in Liu and Vasilyev $(2007)$ that $\phi>\eta_{\text {pen }}$ resulted in the best convergence because the $\phi$ parameter is more forgiving and results in smaller associated errors than with the $\eta_{\text {pen }}$ parameter. For comparison, several cases were tested with $\alpha=1$ and for $\gamma=4.46 \times 10^{-4}$. These results are shown in Figure 8. This shows that the error convergence is not as strong when the $\phi$ and $\eta_{\text {pen }}$ are equal.

\subsection{Practical Implementation}

To set the Brinkman penalization parameters for the shallow water formulation, the procedure is as follows. First, set the sharpness of the Brinkman mask, i.e. the numerical thickness of the smoothed coastal boundary. Ensure that the maximum allowable resolution is sufficient to resolve it. For the Adaptive Wavelet Collocation Method, five to ten points is enough to resolve $\delta_{\text {pen }}$. Assuming the sea surface height initial conditions are known, and therefore, the length scale of the problem is known, $\gamma=\delta_{\text {pen }} / \sqrt{\delta_{\eta}}$ can be calculated. Using Figure 7 as a guideline, the optimal $\phi$ can be estimated. To get $\eta_{\text {pen }}$, maintaining the ratio, $\alpha=\eta_{\text {pen }} / \phi=10^{-2}$, is best for minimizing error (not to mention, this ratio has been tested extensively). However, $\eta_{\text {pen }}<\phi$ will give 


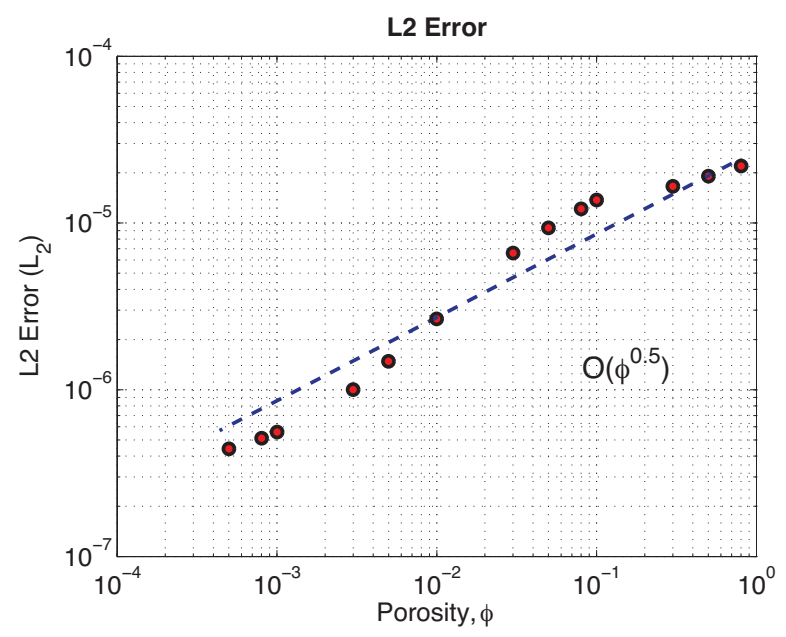

Fig. 8 Plot of L2 error versus porosity parameter, $\phi$ for $\alpha=1$ and for $\gamma=4.46 \times 10^{-4}$

excellent Brinkman results. Although there are various ways to set up these parameters, this procedure serves as a general guideline to pick parameters in a way that will minimize errors.

\subsection{Cost of Brinkman Penalization}

There is added cost associated with the implementation of Brinkman penalization. Depending on the $\delta_{\text {pen }}$ used, the computational cost to resolve the Brinkman boundary may be higher than a conventional boundary wall. This is unavoidable but does contribute to improving the accuracy at the boundaries, so in many cases it is well worth it. The other aspect of Brinkman penalization is the additional domain space. Adding a Brinkman zone means the computational domain needs to be larger, which also increases the computational cost. However, this can easily be minimized by making the zone as small as possible. It is not cost effective to use Brinkman penalization to define straight boundaries. It is a technique to accurately represent complex, variable geometry boundaries, in which case the added computational cost should be expected.

4.5 Two Dimensional Wind-Driven Double Gyre with Brinkman Penalization

Various two dimensional studies have verified the new formulation of Brinkman penalization. The first case is a $2 \mathrm{D}$ wind-driven double gyre test case in a rectangular domain. This test case was solved using the Adaptive Wavelet Collocation Method with the Brinkman penalization method used for flat bottom 
rectangular domain. The results using Brinkman were compared to the same test case solved using the Adaptive Wavelet Collocation Method but applying conventional no-slip boundary conditions on the side walls. Although no-slip boundary conditions are not always used in Munk gyre problems, it has been done extensively (Fox-Kemper and Pedlosky 2004; Fox-Kemper 2004). It is well known that at higher Reynolds number, subtle variations in viscous treatments near the boundaries can strongly affect the basin-wide flow (Fox-Kemper and Pedlosky 2004; Fox-Kemper 2004). Direct comparison to the quasi-geostrophic simulations in those papers with the Brinkman method is underway. Lastly, analytic solutions similar to this test case are given in Pedlosky (1996), however, those solutions are linear and quasi-geostrophic, not the nonlinear shallow water solution presented here. Therefore, comparison to numerical boundary conditions is simpler. These two results show qualitative agreement, as can be seen in Figure 9.

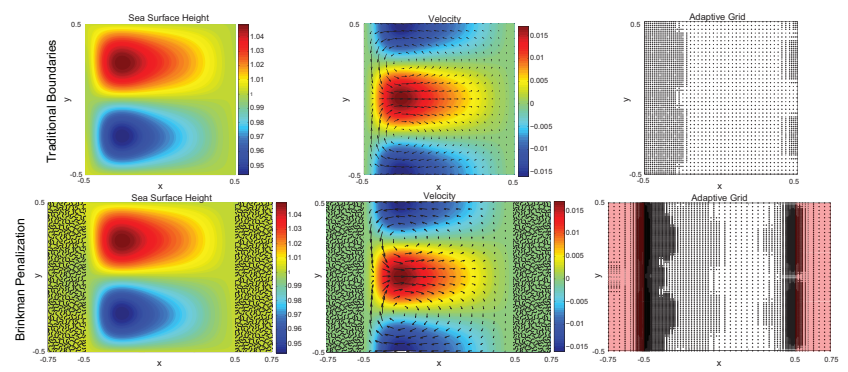

Fig. $92 \mathrm{D}$ plots of sea surface height, velocity, and the adaptive grid for a wind-driven double gyre circulation using Brinkman penalization.

A complete two dimensional convergence study is not necessary, since a thorough $1 \mathrm{D}$ convergence study has been completed. However, to verify that $2 \mathrm{D}$ convergence is similar, a small range of parameters are tested using a $2 \mathrm{D}$ wind-driven single gyre test case. Figure 10 shows that the convergence is slightly less than $O(\phi)$.

Studies of variable bathymetry cannot be compared to conventional boundary conditions, but can verify the robustness of the method. There is a short transient time for this steady state solution to adjust to the Brinkman penalization. This is the case for a rectangular domain, as well. It takes the solution slightly longer to adjust for a non-rectangular domain, but still on a time scale much shorter than the time scale of the problem. With better initial conditions, this can be even further avoided. For boundary currents, it is the steady state solution that is of interest, so it is even less of an issue. Figure 11 shows the solution and grid for a wind-drive gyre in a non-rectangular domain. The grid shows that the method is not only adapting the boundary region, but is also adapting to the dominating circulating gyre structure. 


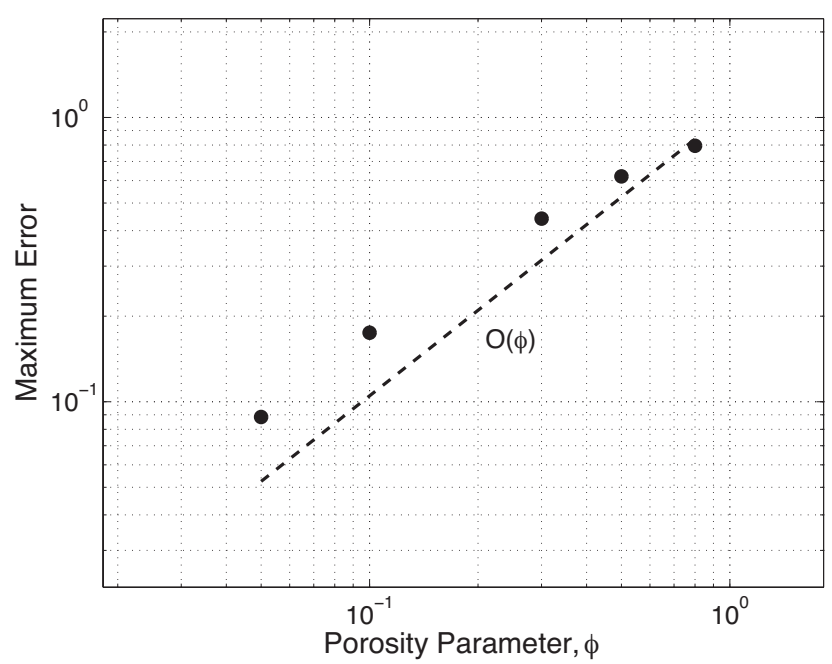

Fig. $102 \mathrm{D}$ convergence study showing max error versus porosity parameter for $2 \mathrm{D}$ winddriven single gyre in a square basin. The dotted line shows $O(\phi)$ convergence, for comparison.
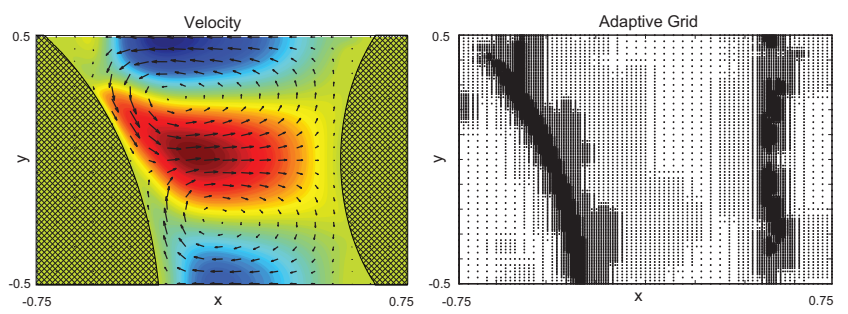

Fig. 11 2D plots of velocity and the adaptive grid for a wind-driven double gyre circulation using Brinkman penalization for variable bathymetry.

\section{Volume Penalization for Modeling Bathymetry}

The ocean bathymetry is a highly varying, intricate, and complex surface. Using current techniques for representation of this bottom boundary results in a surface that is either too crude (stair step representation) or too expensive (body-fitted meshes). The incompressible formulation of Brinkman penalization (Arquis and Caltagirone 1984) is implemented by adding the term, $-\left(\chi / \eta_{\text {pen }}\right) \mathbf{u}$, to the momentum equations. When applied to the hydrostatic primitive equations, the following equations result,

$$
\frac{\partial \eta}{\partial t}+\left(\left.\mathbf{u}_{\mathbf{h}}\right|_{\mathrm{z}=\mathrm{z}_{\max }}\right) \cdot \nabla_{\mathrm{h}} \eta=\left(\left.w\right|_{\mathrm{z}=\mathrm{z}_{\max }}\right) \text {, }
$$




$$
\begin{gathered}
\frac{\partial \mathbf{u}_{h}}{\partial t}+\mathbf{u} \cdot \nabla \mathbf{u}_{h}+\frac{1}{R o} f \hat{\mathbf{k}} \times \mathbf{u}_{h}=-\nabla_{h} P+\frac{1}{R e} \nabla^{2} \mathbf{u}_{h}-\frac{\chi}{\eta_{\mathrm{pen}}} \mathbf{u} \\
\frac{\partial w}{\partial z}=-\nabla_{\mathrm{h}} \mathbf{u}_{\mathbf{h}},\left(\left.w\right|_{\mathrm{z}=\mathrm{z}_{\mathrm{bottom}}}=0\right) \\
\frac{\partial P}{\partial z}=-\frac{1}{F r^{2}},\left(\left.P\right|_{\mathrm{z}=\mathrm{z}_{\max }}=\frac{\eta}{F r^{2}}\right)
\end{gathered}
$$

The added Brinkman term forces the velocity to be zero in the Brinkman zone. As a first attempt to utilize volume penalization to represent ocean boundaries, Brinkman penalization for no-slip boundary conditions is used as a benchmark case. Results are shown in Figure 12 compared against no-slip wall conditions directly applied to the boundaries.
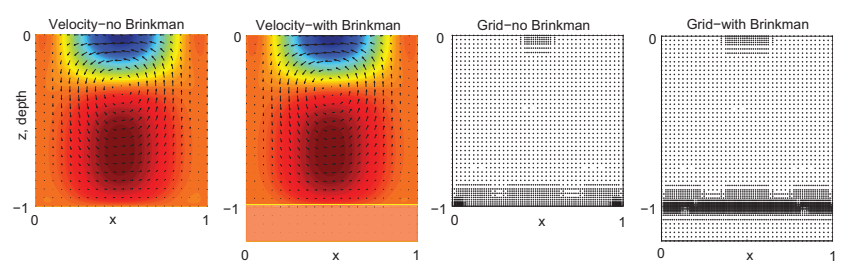

Fig. 12 Demonstration of no-slip boundary conditions with and without Brinkman penalization. The test case shown here is a constant horizontal wind force on the top surface (decaying exponentially downward) with no-slip boundary conditions on the sidewalls, which creates an $\mathrm{x}-\mathrm{z}$ circulation pattern convenient for testing bottom boundary conditions.

In addition, the convergence of the no-slip Brinkman penalization is verified. Figure 13 shows the strong error convergence with decreasing penalization parameter.

Comparison of the solution profiles demonstrates accurate representation of no-slip conditions using this method. However, for the large scale ocean modeling of interest, no-slip boundary conditions are not necessary or realistic. To avoid resolving the boundary layer associated with no-slip conditions, it is convenient to extend this methodology to slip conditions, $\partial u / \partial z=\kappa u$. This boundary condition is somewhat more general than a strict slip boundary condition of $\partial u / \partial z=0$. However, the latter would require us to impose an additional boundary layer drag parameterization such as Ekman layer drag (e.g., Pedlosky 1987, chp. 4.5) or quadratic drag (e.g., Arbic and Scott 2008); the form used here is a simple step in that direction. The idea behind this method is very similar to the no-slip case. The term added has a time scale much smaller than the time scale of the problem, so the boundary conditions are applied on this small time scale, $\eta_{\text {pen }}$.

Two different volume penalization methods are developed and tested. The full set of penalized governing equations are

$$
\frac{\partial \eta}{\partial t}+\left(\left.\mathbf{u}_{h}\right|_{\mathrm{z}=\mathrm{z}_{\max }}\right) \cdot \nabla_{h} \eta=\left(\left.w\right|_{\mathrm{z}=\mathrm{z}_{\max }}\right),
$$




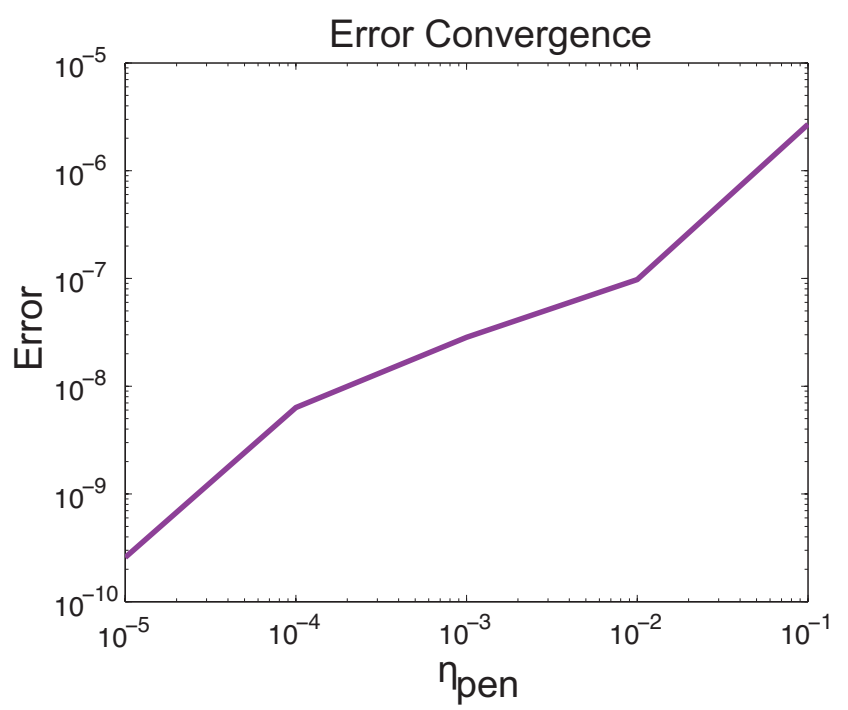

Fig. 13 A plot of error convergence for decreasing penalization parameter for no-slip conditions..

(a)

$\frac{\partial \mathbf{u}_{h}}{\partial t}+\mathbf{u} \cdot \nabla \mathbf{u}_{h}+\frac{1}{R o} f \hat{\mathbf{k}} \times \mathbf{u}_{h}=-\nabla_{h} P+\frac{1}{R e} \nabla^{2} \mathbf{u}_{h}+\frac{\chi}{\eta_{\text {pen }}}\left(-\kappa \frac{\partial \mathbf{u}_{h}}{\partial z}+\frac{\partial^{2} \mathbf{u}_{\mathbf{h}}}{\partial z^{2}}\right)$,

(b)

$$
\begin{gathered}
\frac{\partial \mathbf{u}_{h}}{\partial t}+\mathbf{u} \cdot \nabla \mathbf{u}_{h}+\frac{1}{R o} f \hat{\mathbf{k}} \times \mathbf{u}_{h}=-\nabla_{h} P+\frac{1}{R e} \nabla^{2} \mathbf{u}_{h}+\frac{\chi}{\eta_{\text {pen }}}\left(-\kappa^{2} \mathbf{u}_{h}+\frac{\partial^{2} \mathbf{u}_{\mathbf{h}}}{\partial z^{2}}\right), \\
\frac{\partial w}{\partial z}=-\nabla_{h} \mathbf{u}_{h},\left(\left.w\right|_{\mathrm{z}=\mathrm{z}_{\text {bottom }}\left(\mathbf{x}_{\mathbf{h}}\right)}\right)=0 \\
\frac{\partial P}{\partial z}=-\frac{1}{F r^{2}},\left(\left.P\right|_{\mathrm{z}=\mathrm{z}_{\text {max }}}=\frac{\eta}{F r^{2}}\right),
\end{gathered}
$$

where (a) and (b) are two different volume penalization options.

For the flat bottom test problem, both methods converge equally well. The test used is a wind forced circulation in the zonal and depth direction. The wind forces only the top surface, but there is a smooth transition to zero through the top half of the domain to avoid numerical instability. This case demonstrates the advantage of slip conditions, while also showing what slip conditions look like at the boundary for a simple circulation problem. The velocity profile is extremely sensitive to the value of $\kappa$. Figures 14 and 15 show plots of velocity and the adaptive grid for both $\kappa=1$ and $\kappa=10$. Additionally, 
as a result of the steeper slope in a higher $\kappa$ value, there is a finer resolution near the penalization zone.
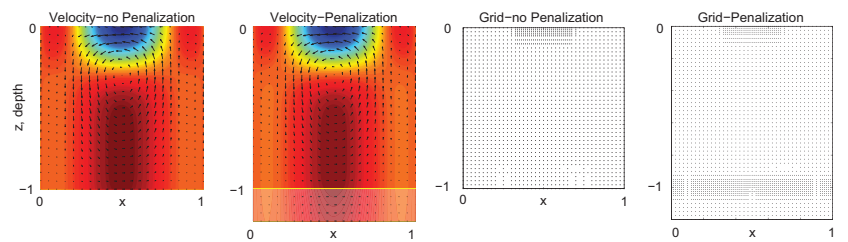

Fig. 14 Demonstration of slip boundary conditions with and without Brinkman penalization for $\kappa=1$.
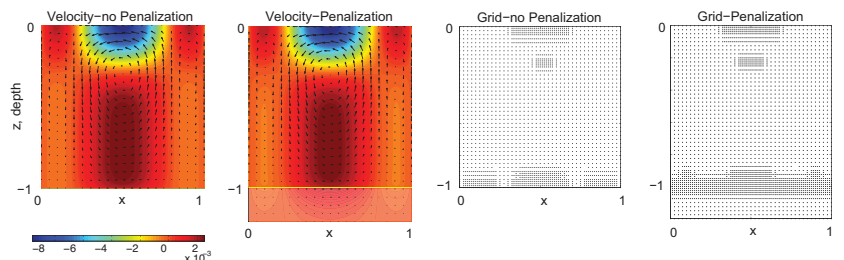

Fig. 15 Demonstration of slip boundary conditions with and without Brinkman penalization for $\kappa=10$.

This new volume penalization formulation enforces slip boundary conditions. The advantage of such a formulation is the computational savings associated with not needing to resolve the boundary layer at the boundaries, which is necessary with the conventional Brinkman penalization no-slip formulation. This same volume penalization has also been applied to the non-hydrostatic equations and initial testing has indicated that it performs similarly.

\section{Concluding Remarks}

Various formulations of Brinkman penalization has been developed and tested, all solved using the Adaptive Wavelet Collocation Method. The shallow water formulation of Brinkman penalization has been thoroughly discussed such that applying it to new and different ocean problems using any numerical method can be done. The key to successfully using this penalization is to carefully and intentionally choose all numerical parameters, as discussed in this paper. Even though Brinkman penalization can be used in combination with any numerical methodology, the use of adaptive mesh refinement techniques such as the wavelet collocation method considerably improves its versatility 
and robustness, mainly due to the completely automated definition and adequate resolution of complex geometry. Lastly, one particular advantage of Brinkman penalization is the ability to represent not only complex, but evolving bathymetry.

The preliminary work on volume penalization for applying slip conditions at the bottom bathymetry boundary for use in the non-hydrostatic and hydrostatic primitive equations was also presented. Further work includes generalizing this to a normal derivative condition rather than a vertical derivative condition, as well as testing in three dimensions.

Acknowledgements This work was supported by DOE-CCPP (DE-FG02-07ER64468). BFK was supported by NSF FRG 0855010. Also, thank you to Scott Reckinger.

\section{References}

Adcroft A, Marshall J (1998) How slippery are piecewise-constant coastlines in numerical ocean models? Tellus A 50(1):95-108

Adcroft A, Hill C, Marshall J (1997) Representation of topography by shaved cells in a height coordinate ocean model. Mon Weather Rev 125(2293-315)

Angot P (1999) Analysis of singular perturbations on the Brinkman problem for fictitious domain models of viscous flows. Math Method Appl Sci 22:1395-412

Angot P, Bruneau CH, Fabrie P (1999) A penalization method to take into account obstacles in viscous flows. Numer Math 81(497-520)

Arbic B, Scott R (2008) On quadratic bottom drag, geostrophic turbulence, and oceanic mesoscale eddies. Journal of Physical Oceanography 38(1):84103

Arquis E, Caltagirone JP (1984) Sur les conditions hydrodynamiques au voisinage d'une interface milieu fluide - milieu poreux : application à la convection naturelle. C R Acad Sci Paris II 299:1-4

Blackstock DT (2000) Fundamentals of Physical Acoustics. John Willey and Sons, Inc.

Chen C, Liu H, Beardsley RC (2003) An unstructured grid, finite-volume, three-dimensional, primitive equations ocean model: Application to coastal ocean and estuaries. J Atmos Ocean Tech 20(159-86)

Chui C (1997) Wavelets: A Mathematical Tool for Signal Analysis. SIAM Monographs on Mathematical Modeling and Computation, SIAM, Philadelphia

Chung C (1995) Wave propagation and scattering in computational aeroacoustics

Collins WD, Bitz CM, Blackmon ML, Bonan GB, Bretherton CS, Carton JA, Chang P, Doney SC, Hack JJ, Henderson TB, Kiehl JT, Large WG, McKenna DS, Santer BD, Smith RD (2006) The Community Climate System Model version 3 (CCSM3). J Clim 19:2122-43 
Danilov S, Kivman G, Schroter J (2004) A finite element ocean model: Principles and evaluation. Ocean Model 6(125-50)

Daubechies I (1992) Ten Lectures on Wavelets. No. 61 in CBMS-NSF Series in Applied Mathematics, SIAM, Philadelphia

Donoho DL (1992) Interpolating wavelet transforms. Tech. Rep. 408, Department of Statistics, Stanford University

Fox-Kemper B (2004) Wind-driven barotropic gyre II: Effects of eddies and low interior viscosity. J Mar Res 62:195-232

Fox-Kemper B, Pedlosky J (2004) Wind-driven barotropic gyre I: Circulation control by eddy vorticity fluxes to an enhanced removal region. J Mar Res 62:169-93

Fringer OB, Gerritsen M, Street RL (2006) An unstructured-grid, finitevolume, nonhydrostatic, parallel coastal ocean simulator. Ocean Model 14(139-73)

Herrnstein A, Wickett M, Rodrigue G (2005) Structured adaptive mesh refinement using leapfrog time integration on a staggered grid for ocean models. Ocean Model 9:283304

Iskandarani M, Haidvogel DB, Levin J (2003) A three-dimensional spectral element model for the solution of the hydrostatic primitive equations. J of Comput Phys 2(361-705)

Kevlahan KR, Vasilyev OV (2005) An adaptive wavelet collocation method for fluid-structure interaction at high reynolds numbers. SIAM J Sci Comput 26:1894-1915

Kevlahan KRN, Vasilyev OV, Cherhabili A (2000) An adaptive wavelet method for turbulence in complex geometries. In: Deville M, Owens R (eds) 16th Imacs World Congress 2000, Proceedings, Lausanne - August 21-25, 2000, IMACS, vol 411-39

Kevlahan NKR, Alam JM, Vasilyev OV (2007) Scaling of space-time modes with Reynolds number in two-dimensional turbulence. J Fluid Mech 570:217-26

Liandrat J, Tchamitchian P (1990) Resolution of the 1d regularized Burgers equation using a spatial wavelet approximation. Tech. rep., NASA Contractor Report 187480, ICASE Report 90-83, NASA Langley Research Center, Hampton VA 23665-5225

Liu Q, Vasilyev OV (2007) A Brinkman penalization method for compressible flow in complex geometries. J Comput Phys 227:946-66

Lynch DR, Ip JTC, Naimie CE, Werner FE (1996) Comprehensive coastal circulation model with application to Gulf of Maine. Cont Shelf Res 16(875906)

Mallat S (1998) A Wavelet Tour of Signal Processing. Academic Press

Marshall J, Adcroft A, Hill C, Perelman L, Heisey C (1997) A finite-volume, incompressible Navier-Stokes model for studies of the ocean on parallel computer. J Geophysl Res 102(C3):5753-66

Mittal R, Iaccarino G (2005) Immersed boundary methods. Annu Rev Fluid Mech 37:239-61 
Pain CC, Piggott MD, Goddard AJH, Fang F, Gorman GJ, Marshall DP, Eaton MD, Power PW, de Oliveira CRE (2005) Three-dimensional unstructured mesh ocean modelling. Ocean Model 10(5-33)

Pedlosky J (1987) Geophysical fluid dynamics. Springer

Pedlosky J (1996) Ocean Circulation Theory

Peskin CS (2002) The immersed boundary method. Acta Numerica pp 479-517

Popinet S, Rickard G (2007) A tree-based solver for adaptive ocean modelling. Ocean Model 16(224-49)

Reckinger SJ, Livescu D, Vasilyev OV (2010) Adaptive wavelet collocation method simulations of Rayleigh-Taylor instability. Physica Scripta T142(014064):1-6

Regele JD, Vasilyev OV (2009) An adaptive wavelet-collocation method for shock computations. Int J Comp Fluid Dyn 23(7):503-18

Schneider K, Vasilyev OV (2010) Wavelet methods in computational fluid dynamics. Ann Rev Fluid Mech 42:473-503

Sweldens W (1998) The lifting scheme: A construction of second generation wavelets. SIAM J Math Anal 29(2):511-46

Tseng YH, Fersiger JH (2003) A ghost-cell immersed boundary method for flow in complex geometry. J of Comput Phys 192(593-623)

Vasilyev OV (2003) Solving multi-dimensional evolution problems with localized structures using second generation wavelets. Int J Comput Fluid Dyn $17(2): 151-68$

Vasilyev OV, Bowman C (2000) Second generation wavelet collocation method for the solution of partial differential equations. J Comput Phys 165:660-93

Vasilyev OV, Kevlahan NK (2005) An adaptive multilevel wavelet collocation method for elliptic problems. J Comp Phys 206(2):412-31

Vasilyev OV, Kevlahan NKR (2002) Hybrid wavelet collocation - Brinkman penalization method for complex geometry flows. Int J Numer Meth Fl 40:531-38

Vasilyev OV, Yuen DA, Paolucci S (1997) The solution of PDEs using wavelets. Computers in Phys 11(5):429-35

White L (2007) Accuracy and consistency in finite element ocean modeling. $\mathrm{PhD}$ thesis, Universite Catholique De Louvain 